\title{
ORIENTAÇÃO PARA O MERCADO: ORIGENS, AVANÇOS E PERSPECTIVAS
}

\author{
André Torres Urdan \\ Professor da FGV-EAESP \\ E-mail: aturdan@fgvsp.br \\ Angela da Rocha \\ Professora da UFRJ-Coppead \\ E-mail: angela@coppead.ufrj.br
}

A RAE, dando continuidade à série "RAE-clássicos", propiciará aos leitores em língua portuguesa, nesta edição e nas próximas três, alguns trabalhos seminais do pensamento de marketing publicados originalmente em inglês. Nesta edição são publicados dois artigos que abriram uma nova direção de pesquisa, referente à orientação das empresas para o mercado. O texto a seguir apresenta a inserção dessa iniciativa na evolução da disciplina de Marketing, bem como discute os seus avanços até o momento atual (inclusive as repercussões no Brasil) e suas perspectivas.

\section{SURGIMENTO DA ORIENTAÇÃO PARA 0 MERCADO NA TEORIA DE MARKETING}

Os acadêmicos de Marketing que antecederam a Segunda Guerra Mundial estavam preocupados com a definição das atividades de marketing e a estrutura e o funcionamento dos mercados. Mas a complexidade das mudanças no pós-guerra, aliada à expansão das empresas norte-americanas no exterior, deslocou o interesse acadêmico. A chamada Escola de Pensamento Gerencial de expoentes como Neil Borden, Jerome McCarthy e Theodore Levitt - materializou uma teoria de marketing de cunho gerencial, com contribuições que frutificam até hoje (Sheth, Gardner e Garrett, 1988). Nessa linha, em um dos primeiros esforços de síntese do conhecimento acumulado, em 1965, Michael Halbert su- geria como tarefa do cientista sistematizar e testar as teorias de marketing implícitas na atuação dos executivos. Defendia uma teorização voltada para o decisor e seus problemas: "qualquer noção adequada de teoria deve envolver o comportamento de pessoas que estão operando com a classe de fenômenos sobre a qual ela foi formulada" (Halbert, 1965, p. 5).

Mais atrás, diversos autores enfatizaram a importância de a empresa servir o mercado (por exemplo, Converse e Huegy, 1946). Indo além, McKitterick (1957) deu realce a uma filosofia organizacional centrada no entendimento e atendimento das necessidades e dos desejos dos consumidores, que chamou de "conceito de gerência de marketing". A idéia de que as empresas, para sua sobrevivência e sucesso, teriam de orientar-se para o mercado difundiu-se com o manifesto "Miopia em marketing", de Theodore Levitt, publicado em 1960 na Harvard Business Review. Ele mostrava que as empresas fracassavam por não focar a compreensão das expectativas dos clientes e a forma como diferentes produtos vão se sucedendo no atendimento dessas necessidades. Impunha-se uma "orientação para o cliente" para que as empresas aprendessem a ver como função primordial "não a produção de bens e serviços, mas a aquisição de clientes" (Levitt, 1960, p. 56). Por volta de 1965 , a maior parte dos livrostexto já incorporava o "novo conceito de marketing", colocando o consumidor no centro da gestão (Bell e Emory, 1971). 
Todavia, o conceito de marketing parecia muito menos aplicado nas empresas (Pelham e Wilson, 1996). Day (1994) identificou, nesse ponto, um paradoxo. Ainda que, por décadas, profissionais tivessem sido chamados a "permanecer junto ao cliente", a "colocar o consumidor no topo do organograma" e a reconhecer como essencial criar e reter clientes satisfeitos, o conceito de marketing persistia mais como matéria de fé do que base prática para a administração empresarial. Para explicar e resolver o desencontro, tomou vulto o estudo da orientação para o mercado, dada a ausência, na literatura de marketing, de esquemas sintéticos e acessíveis para implementar esse conceito.

Também urgia demonstrar o presumido efeito positivo de uma filosofia de marketing sobre os resultados obtidos pelas empresas. A importância atribuída ao conceito de marketing vinculava-se à percepção de que a empresa efetivamente orientada para o mercado alcançaria desempenho superior. Kotler (1972, p. 23) apontava tal orientação como elemento-chave para a organização atingir seus objetivos. Essa melhora do desempenho viria da maior eficiência em custos e investimento, satisfação dos empregados, preços mais altos, crescimento da receita e potencial de preempção competitiva, tudo isso incrementando os lucros (Day, 1999). Não obstante, a relação entre orientação para o mercado e desempenho empresarial carecia, até fins da década de 1980, de exame sistemático.

A partir de fins da década de 1980, a orientação para o mercado foi sendo tomada como elemento da estratégia empresarial. Day $(1989,1990,1999)$ advogou a relevância de a empresa ser orientada de fora para dentro - a partir do mercado - para construir vantagem competitiva. Para ele, embora outras fontes dessa vantagem estivessem presentes nas firmas em posição superior, elas "superariam suas rivais em muitos ou quase todos os elementos de uma orientação para o mercado" (Day, 1999, p. 21).

Então uma onda de pesquisas sobre orientação para o mercado se formou nos Estados Unidos no início da década de 1990 e propagou-se pelo mundo acadêmico. A impulsioná-la estavam os artigos de Narver e Slater (1990) e Jaworski e Kohli (1993), traduzidos nesta edição da RAE. Eles objetivavam, no plano conceitual e operacional, definir e, na realidade empírica, investigar o construto orientação para o mercado, avaliando seus antecedentes e conseqüências. Desde então, diversos esforços teóricos, metodológicos e empíricos foram empreendidos em torno do tema, tornando-o central no marketing estratégico (Steinman, Deshpandé e Farley, 2000).

\section{CONCEPÇÕES E MENSURAÇÃO DA ORIENTAÇÃO PARA $O$ MERCADO}

Até a década de 1990 não se encontravam disponíveis escalas adequadas para medir a orientação para o mercado de uma empresa, à exceção de esforços pontuais. Uma das poucas investidas foi a de Griffin (1982), que avaliou o grau de associação entre o uso de "métodos modernos de marketing" e o sucesso empresarial. Elaborou um instrumento de medida com quatro fatores: bases de conhecimento de marketing, planejamento de marketing, execução e avaliação e controle. A escala continha elementos de orientação para o mercado, embora também utilizasse variáveis descritivas do uso dos "métodos". Com ela, Griffin (1982) encontrou suporte empírico para sua hipótese, mas Silva (1989), utilizando a escala com corretoras de valores no Brasil, não constatou a mesma relação.

Foi a partir dos dois trabalhos destacados nesta $R A E$ - ambos originalmente publicados no Journal of Marketing - que a mensuração da orientação para o mercado avançou substancialmente. No de Narver e Slater (1990), a orientação para o mercado é delineada com cinco elementos: três componentes comportamentais (orientação para o cliente, orientação para o concorrente e coordenação interfuncional) e dois critérios de decisão (foco no longo prazo e rentabilidade). O procedimento de geração da escala está cuidadosamente descrito no artigo, embora a íntegra da escala dele não faça parte. Os autores propuseram que o construto seria unidimensional, uma vez que os diversos componentes encontravam-se logicamente inter-relacionados. No entanto, quando submetidas ao teste de confiabilidade, as variáveis de desempenho apresentaram baixos índices de confiabilidade, não permitindo concluir pela unidimensionalidade do construto, levando os autores a admitirem que a tentativa de incluir os dois critérios de decisão no construto de orientação para o mercado não havia sido bem-sucedida.

O artigo de Jaworski e Kohli (1993) - o segundo desta edição - envolve a preparação de uma escala de orientação para o mercado. Nisso deve ter ajudado um estudo prévio qualitativo, com a formulação conceitual posteriormente operacionalizada (Kohli e Jaworski, 1990). Em sua concepção, a orientação para o mercado inclui três dimensões: geração de inteligência, disseminação de inteligência e capacidade de resposta. A escala - denominada Markor - é apresentada no artigo, assim como um modelo de antecedentes e conseqüências da orientação para o mercado. A escala 
posteriormente passou de 32 para 20 itens (Kohli, Jaworski e Kumar, 1993) e, como tal, vem sendo amplamente utilizada por pesquisadores. Um refinamento da escala Markor foi proposto por Matsuno, Mentzer e Rentz (2000), mantendo a estrutura tridimensional, mas com 22 itens, que dessa forma atenderiam melhor aos critérios de mensuração.

Além desses dois trabalhos, um recurso prático foi proposto por Day (1999), não para fins de pesquisa, mas para auto-avaliação por executivos do nível de orientação para o mercado. Esse questionário encontra-se transcrito em obra do autor publicada no Brasil (Day, 2001). Ele reconhece cinco componentes na orientação para o mercado: orientação geral (valores, crenças e comportamentos); capacidade de sentir o mercado; capacidade de relacionamento com o mercado; visão estratégica; sistemática organizacional. O questionário de Day foi refinado por pesquisadores brasileiros para uso científico (Mello e Souza, 2003; Souza e Mello, 2003).

De qualquer modo, nada foi mais marcante na mensuração da orientação para o mercado do que as escalas surgidas a partir dos empenhos de Narver e Slater (1990) e Jaworski e Kohli (1993), nos artigos inseridos nesta edição da RAE. De modo geral, nas concepções sobre orientação para o mercado, há semelhanças entre os componentes comportamentais de Narver e Slater (1990) e os de Jaworski e Kohli (1993). Todavia, o primeiro trabalho centra-se em informações, ao passo que o segundo se baseia em ações profissionais.

\section{TESTES EMPÍRICOS DA ORIENTAÇÃO PARA 0 MERCADO}

$\mathrm{Na}$ esteira da vertente fomentada por Narver e Slater (1990) e Jaworski e Kohli (1993), Kirka, Jayachandran e Nearden (2005) realizaram uma meta-análise sobre orientação para o mercado, abrangendo impressionantes 114 artigos empíricos. Em uma meta-análise são agrupadas amostras e resultados de estudos de terceiros, com o propósito de extrair conclusões relativas ao conjunto, com estimativas mais precisas da magnitude e significação das relações entre variáveis. Tratase de ampla revisão sistemática de estudos quantitativos para combinar, sintetizar e integrar as informações neles contidas, com poder aumentado para clarificar o estado de conhecimento sobre uma matéria.

$\mathrm{Na}$ extensa série de estudos prévios, a meta-análise em pauta identificou três principais antecedentes da orientação para o mercado: ênfase da alta administração, dinâmica interdepartamental e sistemas organizacionais. Quanto às conseqüências dessa orientação, quatro foram referidas: desempenho organizacional (geral, lucros, vendas e participação de mercado), consequêencias para o cliente (qualidade, lealdade e satisfação), conseqüências para a inovação (capacidade de inovar, desempenho de novo produto) e conseqüências para os funcionários (comprometimento organizacional, espírito de equipe, orientação para o cliente, conflito de papel e satisfação no cargo). Os moderadores da relação entre orientação para o mercado incluíram turbulência de mercado/ambiental, turbulência tecnológica e intensidade competitiva. As características de mensuração e das amostras incluíam desempenho baseado em custo versus receita, medidas objetivas e subjetivas, medidas com item único versus multiitens, empresas industriais versus de serviços e diferentes contextos culturais.

Ao final do processo meta-analítico realizado por Kirka, Jayachandran e Nearden (2005), foi crucial a conclusão de que a orientação para o mercado exerce, de fato, efeitos positivos significativos sobre o desempenho organizacional e que "embora a implementação de uma orientação para o mercado possa demandar recursos, gera lucros superiores aos custos envolvidos em sua implementação, ao mesmo tempo em que propicia o crescimento das receitas" (Kirka, Jayachandran e Nearden, 2005, p. 37).

\section{ORIENTAÇÃO PARA O MERCADO: O BRASIL E OS ESTUDOS BRASILEIROS}

A prática administrativa calcada em genuína preocupação com o cliente é mais comum em contextos de concorrência acirrada e mercados com expectativas elevadas. No Brasil, eram bem diferentes as circunstâncias até o início da década de 1990. Na maior parte dos setores prevalecia certa acomodação entre os participantes, talvez satisfeitos com os resultados obtidos. Uma excessiva intervenção governamental na economia e proteção contra a concorrência externa reforçavam o quadro de relativa tranqüilidade competitiva para muitas das empresas em operação no país. Paralelamente, os níveis de expectativa e consciência do consumidor brasileiro se encontravam aquém daqueles observados em mercados mais disputados e desenvolvidos. Logo, pode-se cogitar que havia baixo grau de orientação para o mercado entre as empresas no Brasil, e que isso, em uma perspectiva de curto prazo, não constituía problema para elas. 
Esse panorama viria a mudar com certa rapidez, exigindo uma resposta das empresas. No entanto, se a orientação para o mercado é uma cultura, ela não se enraíza em uma companhia rapidamente. Em vez disso, é de se esperar que efeitos residuais do contexto passado, de séculos de práticas orientadas para o vendedor e não para o cliente, ainda estejam presentes.

Nesse panorama, diversos estudos brasileiros versaram sobre orientação para o mercado. Silveira (1998) avaliou a orientação para o mercado entre firmas do setor calçadista; Menna (2001) testou a relação entre orientação para o mercado e desempenho no varejo de confecções, enquanto Silva, Damacena e Melo (2002) abordaram essa relação no setor vinícola; Sampaio, Perin e Henriqson (2004) e Perin e Sampaio (2004) pesquisaram as relações entre orientação para o mercado, orientação para aprendizagem e porte empresarial na indústria eletroeletrônica; e Antoni, Damacena e Lezana (2004) aplicaram uma versão modificada da escala Markor a instituições de ensino superior e relacionaram a orientação para o mercado com o desempenho organizacional. Souza e Mello (2003) e Souza (2004, 2005) refinaram e validaram a escala de Day (1999), associando a medida de orientação para o mercado a variáveis de desempenho e relacionamento com clientes. De modo geral, tanto os trabalhos que utilizaram a escala Markor quanto os que usaram a escala de Day encontraram uma relação positiva entre orientação para o mercado e variáveis de desempenho.

\section{PERSPECTIVAS NA ORIENTAÇÃO PARA O MERCADO}

Olhando para o futuro, algumas perspectivas afiguramse importantes para o avanço do conhecimento sobre orientação para o mercado. Nas pesquisas caberá buscar maior variabilidade, tanto nas variáveis de orientação para o mercado quanto em seus antecedentes e conseqüências. É um requisito, para robustecer as análises estatísticas, reunir uma gama suficientemente grande de empresas que apresentem situações bem distintas nesses construtos.

Caberá superar o viés de desejabilidade social, resultante de respostas que não são aquilo que os respondentes pensam e acreditam ser socialmente apropriado, mas o que eles percebem como tal. A possibilidade desse tipo de viés deve ser sempre considerada quando o fenômeno investigado engloba assuntos sensíveis. Em quase toda parte - da grande imprensa às escolas de Administração - ouve-se um discurso sobre o papel vital dos clientes. Não é diferente o que dizem os execu- tivos em pesquisas que procuram medir a orientação para o mercado. Por conseguinte, é possível que o quadro geral de orientação para o mercado que se capta não seja verdadeiro, sendo a realidade menos favorável do que parece ser. Uma solução será obter informações com outros funcionários das empresas, como os vendedores, e com os próprios clientes.

Um desafio será articular a coleção já considerável de antecedentes, moderadores e conseqüências da orientação para o mercado, que ainda tende a se expandir em vista de prováveis novas descobertas. Mais do que ter esses fatores expostos separadamente ou em algumas poucas relações parciais, o avanço científico exigirá referenciais integrativos. É a chamada elaboração teórica, em que se busca explicar um evento da forma mais abrangente e precisa possível (Selltiz et al., 1976). Em uma teoria, a adição de relações permite dizer mais sobre a matéria, com explicações mais completas. A elaboração de um modelo, como recurso teórico, começa por simplificações, porém a sucessão histórica de modelos tende a ser um progresso em complexidade, no caminho para uma abordagem mais rigorosa de sistemas tomados com limites mais amplos (Mazzon, 1978).

Não poderá faltar a continuidade da perspectiva multidisciplinar na investigação da orientação para o mercado. Tradicionalmente, o estudo de Administração tem-se dado mais dentro dos limites de disciplinas. O pesquisador de marketing restringe suas discussões a outros pesquisadores de marketing, estudiosos de recursos humanos pouco compartilham seus pontos de vista e pesquisadores de finanças atuam sob outros prismas. Entretanto, cada grupo de acadêmicos está contemplando o mesmo "objeto", embora mudando o ângulo. Sendo as organizações sistemas de partes interrelacionadas, não há como compreendê-las estudando as partes isoladamente. Será indispensável contemplar simultaneamente, entre outras funções, marketing e finanças, viabilizando referenciais teóricos mais realistas e poderosos. Também as contribuições provenientes da área de estratégia, em que se tem desenvolvido uma multiplicidade de estudos sobre desempenho organizacional, poderão dar oportunidade à fertilização cruzada, uma das mais importantes fontes de inovação em uma disciplina.

A questão da cultura deverá se revestir de caráter prioritário, dada a concepção de orientação para o mercado como cultura organizacional. Intervém a conjectura de Farley (1997), considerando como problema crucial as possíveis características culturais do próprio marketing e suas fortes raízes com o Ocidente e, sobretudo, com 
os Estados Unidos. A dúvida é se a "tecnologia" do marketing pode ser diretamente transferida para sociedades culturalmente diversas. Impõe-se saber se a cultura nacional brasileira possui traços compatíveis ou facilitadores para a orientação para o mercado. Essa é uma direção geral para pesquisas futuras a merecer reflexão de todos os envolvidos com o marketing no Brasil, especialmente os acadêmicos.

Tudo isso é premente diante do aumento da competição no Brasil em virtude de tendências como maior abertura ao exterior e baixo ritmo de crescimento econômico, tornando as oportunidades mais disputadas. Nessas condições, sobressai a necessidade de estudos sobre orientação para o mercado, seus antecedentes e suas conseqüências. Que venham mais pesquisas sobre esse domínio no Brasil, lançando luzes sobre tais conceitos e suas relações. O desafio para futuros trabalhos é desenvolver tanto uma teoria sólida quanto diretrizes para os praticantes.

\section{REFERÊNCIAS BIBLIOGRÁFICAS}

ANTONI, V. L.; DAMACENA, C.; LEZANA, A. G. R. L. Um modelo preditivo de orientação para o mercado: um estudo no contexto do ensino superior brasileiro. In: ENCONTRO NACIONAL DA ASSOCIAÇÃO NACIONAL DOS PROGRAMAS DE PÓS-GRADUAÇÃO E PESQUISA EM ADMINISTRAÇÃO, 28., 2004, Curitiba. Anais. Curitiba: ANPAD, 2004.

BELL, M. L.; EMORY, C. W. The faltering marketing concept. Journal of Marketing, v. 35, n. 4, p. 37-42, 1971.

CONVERSE, P. D.; HUEGY, H. W. The Elements of Marketing. New York: Prentice-Hall, 1946.

DAY, G. Strategic Market Planning: The Pursuit of Competitive Advantage. St. Paul: West Publishing Co., 1984.

DAY, G. Market Driven Strategy: Process for Creating Value. New York: Free Press, 1990.

DAY, G. The capabilities of market-driven organizations. Journal of Marketing, v. 58, n. 4, p. 37-52, 1994.

DAY, G. The Market Driven Organization: Understanding, Attracting, and Keeping Valuable Customers. New York: Free Press, 1999.

DAY, G. A empresa orientada para o mercado: compreender, atrair e manter clientes valiosos. Porto Alegre: Bookman, 2001.

FARLEY, J. U. Looking ahead at the marketplace: it's global and it's changing. In: LEHMANN, D. R.; JOCZ, K. E. Reflections on the Futures of Marketing. Chicago: Marketing Science Institute, 1997. p. 15-35.
GRIFFIN, T. Linking the use of modern marketing methods to company success. The Columbia Journal of World Business, v. 17, n. 3, p. 52-61, 1982.

HALBERT, M. The Meaning and Sources of Marketing Theory. New York: McGraw-Hill, 1965.

JAWORSKI, B. J.; KOHLI, A. K. Market orientation: antecedents and consequences. Journal of Marketing, v. 57, n. 3, p. 53-70, 1993.

KIRCA, A. H.; JAYACHANDRAN, S.; BEARDEN, W. O. Market orientation: a meta-analytic review and assessment of its antecedents and impact on performance. Journal of Marketing, v. 69, n. 2, p. 24-41, 2005.

KOHLI, A. K.; JAWORSKI, B. Market orientation: the construct, research propositions, and managerial implications. Journal of Marketing, v. 54, n. 2, p. 1-18, 1990.

KOHLI, A. K.; JAWORSKI, B.; KUMAR, A. MARKOR: a measure of market orientation. Journal of Marketing Research, v. 30, n. 4, p. 467-477, 1993.

KOTLER, P. Marketing Management: Analysis, Planning and Control. 2. ed. Englewood Cliffs, NJ: Prentice-Hall, 1972.

LEVITT, T. Marketing myopia. Harvard Business Review, v. 38, n. 4, p. 4556,1960

MAZZON, J. A. Formulação de um modelo de avaliação e comparação de modelos em marketing. (Dissertação de Mestrado). São Paulo: Faculdade de Economia, Administração e Contabilidade da Universidade de São Paulo, 1978.

MATSUNO, K.; MENTZER, J. T.; RENTZ, J. O. A refinement and validation of the Markor scale. Journal of the Academy of Marketing Science, v. 28, n. 4, p. 527-539.

MCKITTERICK, J. B. What is the marketing management concept? In: BASS, F. M. (Ed.). The Frontiers of Marketing Thought and Science. Chicago, American Marketing Association, 1957. p. 71-82.

MELLO, S. C. B.; SOUZA, A. C. R. Purificação e validação da escala de orientação para o mercado de George Day. In: ENCONTRO NACIONAL DA ASSOCIAÇÃO NACIONAL DOS PROGRAMAS DE PÓS-GRADUAÇÃO E PESQUISA EM ADMINISTRAÇÃO, 27., 2003, Atibaia. Anais. Atibaia: ANPAD, 2003.

MENNA, H. L. Orientação para o mercado e performance: evidências em empresas gaúchas de varejo de confecções masculinas. In: ENCONTRO NACIONAL DA ASSOCIAÇÃO NACIONAL DOS PROGRAMAS DE PÓSGRADUAÇÃO E PESQUISA EM ADMINISTRAÇÃO, 25., 2001, Campinas. Anais. Campinas: ANPAD, 2001.

NARVER, J. C.; SLATER, S. F. The effect of a market orientation on business profitability. Journal of Marketing, v. 54, n. 4, p. 20-35, 1990.

PELHAM, A. M.; WILSON, D. T. A longitudinal study of the impact of market structure, firm structure, strategy, and market orientation culture on dimensions of small-firm performance. Journal of the Academy of Marketing Science, v. 24, n. 1, p. 27-43, 1996. 
PERIN, M. G.; SAMPAIO, C. H. Orientação empresarial, porte empresarial e performance. Revista de Administração de Empresas, v. 44, n. 3, p. $76-88,2004$

SAMPAIO, C. H.; PERIN, M. G., HENRIQSON, E. Orientação para o mercado, orientação para aprendizagem e porte empresarial como antecedentes das dimensões da performance. In: ENCONTRO NACIONAL DA ASSOCIAÇÃO NACIONAL DOS PROGRAMAS DE PÓS-GRADUAÇÃO E PESQUISA EM ADMINISTRAÇÃO, 28., 2004, Curitiba. Anais. Curitiba: ANPAD, 2004.

SELLTIZ, C.; WRIGHTSMAN, L. S.; COOK, S. W. Research Methods in Social Relations. 3. ed. New York: Holt, Rinehart and Winston, 1976.

SHETH, J. N.; GARDNER, D. M.; GARRETT, D. E. Marketing Theory: Evolution and Evaluation. New York: John Wiley \& Sons, 1988

SILVA, R. M. Marketing de serviços: métodos modernos e sucesso nas corretoras da BVRJ. (Dissertação de Mestrado). Rio de Janeiro, Coppead/UFRJ, 1989

SILVA, F. A. C.; DAMACENA, C.; MELO, L. M. R. Orientação para o mercado e performance organizacional: um estudo na indústria vinícola do Rio Grande do Sul. CONGRESSO COPPEAD DE ADMINISTRAÇÃO, 9. 2002, Rio de Janeiro. Anais. Coppead/UFRJ, 2002.
SILVEIRA, T. Verificação do grau de orientação para o mercado em empresas calçadistas do vale dos Sinos. In: ENCONTRO NACIONAL DA ASSOCIAÇÃO NACIONAL DOS PROGRAMAS DE PÓS-GRADUAÇÃO E PESQUISA EM ADMINISTRAÇÃO, 22., 1998, Foz do Iguaçu. Anais. Foz do Iguaçu: ANPAD, 1998.

SOUZA, A. C. R. A orientação para o mercado, o relacionamento com os clientes e o desempenho das empresas: um levantamento junto a empresas que atuam na região Nordeste do Brasil. In: ENCONTRO NACIONAL DA ASSOCIAÇÃO NACIONAL DOS PROGRAMAS DE PÓS-GRADUAÇÃO E PESQUISA EM ADMINISTRAÇÃO, 28., 2004, Curitiba. Anais. Curitiba: ANPAD, 2004.

SOUZA, A. C. R. Estratégia de orientação para o mercado e relacionamento com o cliente em médias e grandes empresas: uma análise de correlação canônica. In: ENCONTRO NACIONAL DA ASSOCIAÇÃO NACIONAL DOS PROGRAMAS DE PÓS-GRADUAÇÃO E PESQUISA EM ADMINISTRAÇÃO, 29., 2005, Brasília. Anais. Brasília: ANPAD, 2005.

SOUZA, A. C. R.; MELLO, S. C. B. Uma avaliação das dimensões da escala de orientação para o mercado de George Day. ENCONTRO DE ESTUDOS EM ESTRATÉGIA - 3 ES, 1., 2003, Curitiba. Anais. Curitiba: ANPAD, 2003.

STEINMAN, C.; DESHPANDÉ, R; FARLEY, J. U. Beyond market orientation: when customers and suppliers disagree. Journal of the Academy of Marketing Science, v. 28, n. 1, p. 109-119, 2000.

\section{Artigo convidado. Aprovado em 21.03.2006}

